Meta

Journal des traducteurs

Translators' Journal

\title{
Aspects méthodologiques de l'évaluation de la qualité du travail en interprétation simultanée
}

\section{Daniel Gile}

Volume 28, numéro 3, septembre 1983

URI : https://id.erudit.org/iderudit/002899ar

DOI : https://doi.org/10.7202/002899ar

Aller au sommaire du numéro

Éditeur(s)

Les Presses de l'Université de Montréal

ISSN

0026-0452 (imprimé)

1492-1421 (numérique)

Découvrir la revue

Citer cet article

Gile, D. (1983). Aspects méthodologiques de l'évaluation de la qualité du travail en interprétation simultanée. Meta, 28(3), 236-243.

https://doi.org/10.7202/002899ar d'utilisation que vous pouvez consulter en ligne. 


\section{ASPECTS MÉTHODOLOGIQUES DE L'ÉVALUATION DE LA QUALITÉ DU TRAVAIL EN INTERPRÉTATION SIMULTANÉE}

Daniel GiLe

\section{INTRODUCTION}

À l'heure actuelle, il n'existe pas de définition fonctionnelle, agréée par les interprètes ou les utilisateurs de leurs services, qui détermine formellement la qualité du travail en interprétation. Les jugements portés par les uns et les autres sont intuitifs, personnels, et ne se réfèrent pas à des critères objectifs.

Compte tenu des combinaisons linguistiques, des dates de disponibilité des interprètes et des considérations personnelles susceptibles d'intervenir, ces jugements semblent suffire aux recruteurs pour la constitution des équipes.

Les efforts des chercheurs gagneraient toutefois à s'appuyer sur une définition fonctionnelle objective et précise. Celle-ci permettrait en effet la réalisation d'analyses fines par rapport à un repère fixe ainsi que la mise en évidence des mécanismes d'interaction entre les variables et paramètres en présence. Les résultats pourraient par la suite servir de base à l'élaboration de propositions pour l'amélioration des méthodes de formation et de travail.

Par ailleurs, la présentation d'une définition descriptive et normative d'une qualité de travail réalisable dans des conditions déterminées est susceptible d'orienter les utilisateurs et de favoriser l'acceptation des règles professionnelles élaborées par l'AIIC, déclenchant ainsi une dynamique d'amélioration générale du niveau de l'interprétation de conférence.

Le présent article vise à mettre en évidence les principales questions fondamentales associées à la notion de qualité du travail en interprétation et à esquisser une démarche pratique de recherche.

\section{LES DEUX FACETTES DU MESSAGE VERBAL}

En situation de communication, l'émission d'un message a une fonction, simple ou multiple. Le locuteur cherche à exposer, à expliquer, à illustrer, à convaincre, à briller, à s'acquitter d'une obligation officielle. Il considère que l'acte de communication est réussi s'il a atteint ses objectifs.

L'effet potentiel du message verbal sur son destinataire repose notamment sur - son contenu informationnel

- sa présentation 
L'importance relative de chacune de ces facettes est variable selon le type de message et la situation. On peut évoquer à titre d'illustration deux exemples très différents, la communication d'un numéro de téléphone par une standardiste et la récitation d'un poème par un amoureux (la récitation du même poème par la standardiste n'aura pas le même effet, comme le démontre avec humour un célèbre sketch d'Yves Montand).

En conférence, les discours de bienvenue et de remerciement sont davantage tributaires de la forme que les discours techniques et scientifiques, dans lesquels c'est le contenu informationnel qui prédomine.

L'efficacité d'une intervention dépend de son contenu informationnel et de sa présentation, mais aussi de leur interaction.

\section{LES PERTES INFORMATIONNELLES DANS LA TRANSMISSION DU MESSAGE}

La permanence du support du message écrit fait que sa réception (la lecture) est :

- itérative : la lecture et l'analyse peuvent être répétées.

- différenciée : on peut choisir de s'attarder sur certains éléments de l'énoncé.

- non linéaire : le lecteur peut revenir en arrière ou avancer à sa guise.

Toutefois, son vocabulaire technique et ses connaissances extra-linguistiques spécialisées sont nettement inférieurs à ceux des destinataires du message, voire très fragmentaires.

En tant que récepteur, l'interprète se trouve donc dans une situation radicalement différente de celle d'un auditeur ordinaire: alors que celui-ci reçoit un message conçu en fonction de ses connaissances, l'interprète se voit en permanence dans l'obligation de suivre un discours technique qui se situe dans un domaine qu'il ne connaît que superficiellement, et qui est destiné à des interlocuteurs dont il ne partage pas le savoir.

C'est pourquoi son interprétation des vibrations sonores captées est moins rapide, moins sûre et plus laborieuse que celle des destinataires réels du message.

À ces difficultés de réception s'ajoutent les contraintes inhérentes à la simultanée : effort de mémoire (les informations reçues doivent être stockées en mémoire pendant un temps plus ou moins long avant d'être restituées dans le discours d'arrivée) et effort de production du discours dans la langue d'arrivée (l'évocation des mots et l'élaboration des phrases ne sont pas toujours spontanées).

C'est pourquoi le travail en cabine demande une concentration intense et continue, qui devient rapidement épuisante. L'accumulation de la fatigue s'accompagne d'une détérioration du discours de l'interprète qui se traduit par une multiplication des contresens, faux-sens, omissions et simplifications, ainsi que par une dégradation de la qualité linguistique de l'interprétation.

Le Code professionnel de l'AIIC contribue à assurer la qualité de la prestation des interprètes par le biais de règles destinées à faciliter la préparation à la conférence, à améliorer les conditions physiques de la communication, et à préserver les interprètes d'une fatigue excessive ${ }^{1}$.

1. Voir notamment l'Article 7 du Code professionnel de l'AIIC. 


\section{FIDÉLITÉ INFORMATIONNELLE DE L'INTERPRÈTE}

Dans des conditions de travail normales (conformes aux critères de l'AIIC), les interprètes sont généralement capables, et il est important de le souligner, de transmettre la totalité des informations pertinentes (les informations non redondantes communiquées intentionnellement) du message de l'orateur ${ }^{2}$.

Toutefois, dans des conditions défavorables, qui sont actuellement la règle plutôt que l'exception, et notamment si les documents de conférence ne sont pas envoyés aux interprètes suffisamment à l'avance, si des orateurs lisent rapidement des textes écrits que l'on n'a pas en cabine, et si la qualité du son est mauvaise, la «capacité» des interprètes est dépassée, ce qui se traduit entre autres par des pertes informationnelles.

Les incidences de ces pertes, dont l'ampleur est très variable, sur l'efficacité du message, sont l'un des principaux déterminants dans l'évaluation de la qualité en interprétation simultanée.

\section{PERTES INFORMATIONNELLES ET EFFICACITÉ DU MESSAGE}

L'existence de pertes informationnelles dans la communication orale directe a été rappelée plus haut. Sans s'attarder sur une comparaison des pertes dans différentes situations, on peut poser la question intéressant directement l'interprétation: des pertes dans le cheminement orateur-délégués et dans l'itinéraire orateur-interprète-délégués, lesquelles portent le plus grand préjudice à l'efficacité du message?

L'interprète est un auditeur professionnel, attentif et intelligent, en ce sens qu'il écoute activement, en recherchant les idées, les enchaînements et les intentions de l'orateur. Il est donc possible, et même probable, qu'il perd moins à l'écoute qu'un auditeur non professionnel.

D'autre part, l'interprète est un orateur que ses aptitudes et sa formation orientent vers un discours clair et cohérent.

À ce propos, une expérience intéressante est celle qui consiste à interpréter alternativement un orateur et son interprète. On constate que l'interprétation est souvent plus facile à comprendre, car elle est moins encombrée de redondances, plus nette et plus clairement articulée dans le raisonnement, reflétant ainsi les efforts d'exégèse de l'interprète.

C'est pourquoi le discours de l'intermédiaire peut s'avérer être en l'occurrence plus compréhensible et plus convaincant que celui de l'orateur en dépit des pertes informationnelles. Une anecdote que l'auteur ne peut authentifier relate l'élection à la présidence d'un comité important d'un délégué connu par ailleurs pour sa médiocrité et son incohérence, à la suite d'une interprétation particulièrement brillante de ses interventions.

Il serait présomptueux d'affirmer que les discours des interprètes sont d'une manière générale plus efficaces que ceux des délégués, qui sont souvent eux-mêmes des orateurs professionnels. Il semble toutefois intéressant de

2. À condition qu'il ne s'agisse pas d'un orateur particulièrement difficile par la rapidité ou la densité de son discours, son accent très marqué, sa mauvaise connaissance de la langue, etc. 
rappeler, à la lumière des observations cì-dessus, qu'en situation de conférence, le passage par l'intermédiaire d'un interprète implique d'une part la possibilité de pertes informationnelles parfois importantes, mais d'autre part une transformation qualitative du discours susceptible de renforcer son impact.

\section{VERS UNE DÉFINITION FONCTIONNELLE DE LA QUALITÉ DU TRAVAIL}

Sous certaines conditions, une fidélité informationnelle «totale» est possible (voir plus haut). Dans ce cas particulier, l'évaluation de la qualité du travail se ramène à l'évaluation de la qualité de la présentation. Dans la pratique, la fréquence et l'étendue des pertes informationnelles sont telles que la fidélité informationnelle devient un paramètre important (et pour certains utilisateurs, exclusif) de la qualité du travail.

L'évaluation se faisant par rapport à un point de référence, il faut préciser que celui-ci n'est pas unique. Pour les interprètes, l'objectif est la fidélité informationnelle totale. Quant aux organisateurs et aux utilisateurs, leurs attentes sont fondées sur leurs contacts passés avec l'interprétation, ou sur ceux de leurs collègues et connaissances.

Les uns n'imaginent pas l'existence des pertes informationnelles. D'autres considèrent que les interprètes, n'étant pas techniciens, ne peuvent comprendre des discours techniques, ni les restituer. Pour eux, l'interprétation est un discours fragmentaire parallèle, à écouter d'une oreille pour s'en servir le cas échéant quand survient une tournure grammaticale difficile dans le discours original. D'autres utilisateurs encore estiment que les interprètes font un discours partiel qui «permet de savoir de quoi il est question» et de dégager les principales idées de l'orateur, mais qui n'est nullement assimilable à l'original.

Il faut reconnaitre que ces différents points de vue se justifient dans la pratique, étant donné la qualité très variable des prestations en conférence, même si les défaillances sont en grande partie imputables à des conditions de travail mauvaises, voire impossibles.

Si les indices de satisfaction sont variables, leur hétérogénéité tient à des différences quantitatives, et non pas qualitatives, ce qui permet l'adoption d'une définition commune : la qualité de l'interprétation est une somme pondérée de la fidélité informationnelle et de la qualité de la présentation du discours de l'interprète, dont les coefficients sont fonction de la nature du message et de la situation.

Soulignons que cette définition porte sur le résultat, et non pas sur le travail même. Les efforts de l'interprète peuvent être d'excellente qualité tout en étant mis en échec par de mauvaises conditions de travail, ce qui d'ailleurs est fréquemment le cas. Néanmoins, l'interprétation a une mission pratique, et pour les utilisateurs, à qui elle est destinée, c'est le résultat qui compte.

\section{LES ÉVALUATEURS}

En conférence, trois catégories d'auditeurs écoutent l'interprétation simultanée: les délégués qui ne comprennent pas la langue de l'orateur, les collègues passifs de l'interprète qui travaille, et ce dernier. 
Les délégués n'écoutent en général que l'orateur ou l'interprète. Bien placés pour juger de la qualité de la présentation et de la cohérence de l'interprétation, ils peuvent difficilement évaluer sa fidélité informationnelle.

Les collègues passifs suivent les débats et écoutent l'interprète actif :

- par curiosité, pour voir «comment il travaille»

- ponctuellement, pour voir comment il surmonte certaines difficultés

- pour l'aider à franchir des obstacles

Ils ont de la fidélité informationnelle de leur collègue actif une idée fondée sur un échantillonnage ponctuel, car il n'est pas possible d'écouter l'intégralité du discours original et de l'interprétation en même temps.

En ce qui concerne la qualité de la présentation, ils sont aussi bien placés que les utilisateurs pour en juger, mais n'ont pas nécessairement les mêmes critères, étant donné leur situation et leurs connaissances différentes.

Quant à l'interprète actif, conscient de certaines pertes informationnelles, il ne les relève pas toutes. Il lui est donc difficile d'évaluer globalement sa fidélité informationnelle. Par ailleurs, absorbé dans une activité complexe, il n'a pas le recul nécessaire pour bien juger la présentation de son discours.

Il semble qu'en conférence, les meilleurs juges de la qualité de l'interprétation soient les interprètes passifs. Cette hypothèse demande néanmoins à être confirmée sur deux plans: vérification de l'exactitude de leur évaluation de la fidélité informationnelle, et vérification de la convergence de leurs critères et de ceux des délégués en matière de qualité de la présentation.

Hors situation, on peut mesurer avec précision la fidélité informationnelle de l'interprétation en comparant les enregistrements de l'original et du discours interprété. Quant à la présentation, elle peut être jugée par toute personne ayant les mêmes critères que les utilisateurs, qui sont les véritables destinataires du message.

\section{PROBLÈMES HUMAINS}

Les interprètes, très conscients de leurs défaillances, sont en outre dans une situation de concurrence économique qui accentue leur vulnérabilité. Ils n'aiment pas être écoutés par des collègues, et certains préfèrent même travailler seuls en cabine et se priver d'un secours possible en cas de «pépin" plutôt que de se sentir jugés par d'autres interprètes.

C'est pourquoi le recrutement de volontaires dans la profession pour des travaux sur la qualité de l'interprétation n'est guère aisé.

Les utilisateurs, quant à eux, s'intéressent plutôt aux orateurs qu'aux interprètes, fréquemment considérés comme un mal nécessaire, onéreux et d'une efficacité douteuse. En conférence, il est difficile d'obtenir la collaboration des délégués, au-delà de quelques commentaires succincts. D'autre part, les volontaires qui se manifestent sont ceux des délégués qui ont été particulièrement impressionnés par le travail des interprètes, ce qui introduit un important biais statistique dans leur évaluation.

Il convient donc d'alléger autant que possible la tâche des délégués en leur demandant peu d'efforts et peu de temps. Une «feuille d'évaluation» bien conçue est peut-être la méthode la plus efficace dont on dispose actuellement. 


\section{ÉVALUATION DE LA FIDÉLITÉ INFORMATIONNELLE}

La fidélité informationnelle est le paramètre le plus facile à évaluer dans l'interprétation. En effet, on distingue aisément des unités d'information circonscrites dans des phrases, des propositions ou des segments d'énoncé plus petits encore. Il est donc possible de cerner avec une assez grande précision les «infidélités», ou «incidents d'interprétation», et de les examiner de près.

Dénombrables, les incidents sont aussi quantifiables, et la détermination des fréquences relatives par types ou par degré de gravité permet de dresser des «cartes topographiques» susceptibles de caractériser des interprètes, des orateurs, des types de discours et des situations.

\section{ÉVALUATION DE LA QUALITÉ DE LA PRÉSENTATION}

La qualité de la présentation du message n'a pas la même réalité «objective» que les «infidélités informationnelles» et ne se laisse pas quantifier avec la même facilité.

En effet, une voix, un ton, un accent qui plaisent aux uns peuvent déplaire à d'autres, et leur importance relative dans la transmission peut varier selon l'auditeur. D'autre part, certaines tournures, certains «tics», certaines fautes de langue ou maladresses irritent plus ou moins.

La réalité d'une définition «objective» de la qualité de la présentation en interprétation est donc conditionnée par la convergence des évaluations subjectives. Les observations préliminaires de l'auteur semblent confirmer l'existence d'une telle convergence sur une échelle ordinale à 7 paliers (qualité très mauvaise, mauvaise, insuffisante, passable, bonne, très bonne, excellente). À mesure que l'analyse s'affine, des divergences apparaissent, mais ne semblent pas affecter l'évaluation globale de la qualité de l'interprétation.

Sous réserve de la confirmation de la convergence de ces tendances dans un échantillon de taille suffisamment grande, l'échelle à 7 paliers apparaît comme un outil adéquat pour l'évaluation de la qualité de la présentation.

\section{ÉVALUATION GLOBALE DE LA QUALITÉ DE L'INTERPRÉTATION}

L'évaluation globale de la qualité de l'interprétation est en même temps un but et un outil : un but, car c'est elle qui détermine en fin de compte la satisfaction des délégués et le choix des recruteurs, et un outil, car elle permet d'évaluer l'importance relative, aux yeux des juges, de la fidélité informationnelle et de la qualité de la présentation.

Les problèmes de quantification sont les mêmes dans l'évaluation globale que dans l'évaluation de la qualité de la présentation, et l'échelle ordinale à 7 paliers lui semble aussi bien adaptée.

Il est d'ailleurs difficile d'obtenir un jugement spontané plus précis que les mentions correspondant aux 7 paliers. L'évaluateur interrogé a bien une "note» générale à donner, mais au-delà, il essaie de faire une synthèse des détails qui l'ont frappé pour moduler son appréciation a posteriori, ce qui est contraire au but recherché. 
L'échelle à 7 paliers ne suffit pas aux besoins de la recherche au-delà d'un stade préliminaire où elle sert d'outil en vue de la détermination de l'importance relative des deux facettes du message dans différentes situations. Par la suite, l'analyse s'affine et se focalise sur des concepts plus précis.

En résumé, il semble que l'évaluation globale de la qualité de l'interprétation ait une réalité professionnelle, ainsi qu'une certaine valeur d'outil à des fins de recherche.

\section{CONTRIBUTION DE L'INTERPRÉTATION CONSÉCUTIVE À L'ÉVALUATION}

Si l'écoute de l'intégralité du discours original et de son interprétation est impossible en simultanée, il n'en est pas de même en consécutive où les délégués ont tout le loisir pour écouter et comparer les deux, à condition de comprendre les deux langues, ce qui est fréquent.

Les délégués, et notamment l'orateur lui-même, sont donc très bien placés pour juger de la qualité de l'interprétation.

Toutefois, compte tenu de certaines différences importantes dans les modalités d'exercice de la consécutive et de la simultanée, les critères de qualité appliqués et applicables ne sont peut-être pas les mêmes.

En matière de présentation, on notera que si la présence de l'interprète est exclusivement acoustique et «électronique» en simultanée, en consécutive, la présence acoustique est naturelle, et il s'y ajoute la présence visuelle.

En ce qui concerne la fidélité informationnelle, compte tenu du fait que de nombreux délégués comprennent souvent le sens général de l'intervention dans l'original et que l'interprétation consécutive allonge les débats (ce qui semble être la cause du recul de ce type d'interprétation, en dépit d'une qualité jugée meilleure qu'en simultanée ${ }^{3}$ ), on préfère fréquemment une bonne synthèse à la reconstitution intégrale du discours.

Ces réserves étant faites, on peut considérer que les convergences une fois cernées, la contribution des évaluateurs de la consécutive sera précieuse, et ce à double titre. D'une part, elle aidera, dans l'absolu, à déterminer l'importance relative de la fidélité informationnelle et de la qualité de la présentation aux yeux d'un jury qui peut «juger sur pièces». D'autre part, elle permettra de comparer l'évaluation de ces juges à celle des utilisateurs de la simultanée, qui n'ont pas les mêmes possibilités.

\section{ORGANISATION GÉNÉRALE DE LA RECHERCHE}

La démarche préconisée par l'auteur se compose de l'exploration de la notion de qualité sur trois plans : la constitution d'un corpus (discours originaux et interprétations), le recueil des évaluations, et l'exploitation des données.

La constitution du corpus est le recueil par enregistrement de discours originaux et de leur interprétation en situation, puis de leur interprétation par des volontaires en laboratoire (avec l'autorisation des organisateurs et des interprètes, et avec la collaboration active d'interprètes volontaires).

3. Voir notamment p. 11 du Bulletin de l'AIIC IX/4, décembre 1981, dans l'article de John Campbell, "Meeting with delegates, a participant's report». 
Il s'agit de constituer un échantillon aussi grand et varié que possible pour permettre de tirer des conclusions solides sur la base d'un corpus représentatif. C'est d'ailleurs la raison pour laquelle seuls des discours «vrais», enregistrés en situation, sont utilisés, à l'exclusion de tout discours fait en laboratoire aux fins de l'expérience.

Les évaluations sont recueillies d'une part sur des «feuilles d'évaluation", en conférence et en laboratoire, et d'autre part lors d'entrevues plus approfondies avec des volontaires.

Quant à l'exploitation des données, elle comporte obligatoirement une composante qualitative d'analyse, mais est essentiellement quantitative: il s'agit de dénombrer les différents phénomènes et évaluations afin d'en faire une analyse statistique, et ce dans le but de cerner des corrélations, des relations causales, des convergences et des divergences, celles-ci, jugées représentatives, pouvant ensuite être analysées qualitativement.

Plutôt que de faire appel à des théories linguistiques et psycholinguistiques pour prévoir ou deviner des phénomènes invisibles ou invérifiables, la présente démarche cherche à faire le point sur ce qui est visible et incontestable avant d'aller plus loin.

Le chercheur isolé atteint vite les limites matérielles de ses possibilités dans cette entreprise qui demande un échantillon vaste et varié. Limites temporelles, d'une part, compte tenu du fait que le nombre d'enregistrements requis se chiffre par dizaines, ce qui représente des centaines d'heures de travail. Limites dans l'accès à des volontaires, également. Enfin, limites dans la variété de conférences et de langues de travail à sa portée.

C'est pourquoi le travail gagnerait en rapidité et en fiabilité à être mené en parallèle par plusieurs chercheurs ou dans plusieurs centres. L'opération ne présente aucune difficulté dans la constitution du corpus, et la mise au point de formules identiques ou compatibles de «feuilles d'évaluation» devrait être relativement simple. Il resterait alors l'organisation des échanges d'informations pour qu'un travail «multi-centres» puisse être mené de manière fructueuse.

L'auteur est à la disposition des chercheurs intéressés. 